\title{
Detection of Francisella tularensis in ticks and identification of their genotypes using multiple-locus variable-number tandem repeat analysis

\author{
Fang Zhang ${ }^{\dagger}$, Wei Liu ${ }^{\dagger 1}$, Xiao-Ming Wu ${ }^{1}$, Zhong-Tao Xin ${ }^{2}$, Qiu-Min Zhao ${ }^{1}$, \\ Hong Yang ${ }^{1}$ and Wu-Chun Cao*1
}

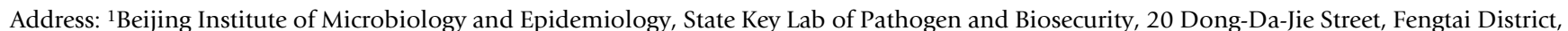
Beijing 100071, PR China and 2Department of Pathology \& Laboratory Medicine, Emory University School of Medicine, Atlanta, GA, USA

Email: Fang Zhang - fang_z760@sohu.com; Wei Liu - liu_weis@sohu.com; Xiao-Ming Wu - wxm@126.com; Zhong-Tao Xin - zxin@emory.edu; Qiu-Min Zhao - zhaoqm@nic.bmi.ac.cn; Hong Yang - anny_hong@163.com; Wu-Chun Cao* - caowc@nic.bmi.ac.cn

* Corresponding author †Equal contributors

Published: 17 September 2008

BMC Microbiology 2008, 8:152 doi:10.1 I86/I47I-2180-8-152
Received: 23 October 2007

Accepted: 17 September 2008

This article is available from: http://www.biomedcentral.com/I47I-2I80/8/I52

(C) 2008 Zhang et al; licensee BioMed Central Ltd.

This is an Open Access article distributed under the terms of the Creative Commons Attribution License (http://creativecommons.org/licenses/by/2.0), which permits unrestricted use, distribution, and reproduction in any medium, provided the original work is properly cited.

\begin{abstract}
Background: Tularemia was reported in China over 50 years ago, however, many epidemical characteristics remain unclear. In the present study, the prevalence of Francisella tularensis in ticks was investigated during an epidemiological surveillance in China and then we measured their genetic diversity by conducting multiple-locus variable- number tandem repeat analysis (MLVA).

Results: 1670 ticks from 2 endemic areas (Inner Mongolia Autonomous Region and Heilongjiang Province) and 2 non-endemic areas (jilin and Fujian Provinces) were collected and tested for evidence of tularemia by nested PCR. The prevalence of Francisella tularensis in ticks averaged $1.98 \%$. The positive rates were significantly different among tick species, with Dermacentor silvarum and Ixodes persulatus responsible for all positive numbers. All $F$. tularensis that were detected in ticks belonged to $F$. tularensis subsp. holarctica and MLVA disclosed genetic diversity. One subtype was identified in 17 of 33 positive tick samples in three different study areas. Another subtype belonging to $F$. tularensis subsp. holarctica genotype was described for the first time in the current study.

Conclusion: The study showed two tick species, D. silvarum and I. persulatus harboring the pathogen of tularemia in natural environment, indicating these two tick species might have a role in tularemia existence in China. MLVA results disclosed the genetic diversity $F$. tularensis and identified one genotype as the most prevalent among the investigated ticks in China.
\end{abstract}

\section{Background}

Tularemia, also known as rabbit fever or deer-fly fever, is caused by the gram-negative intracellular pathogen Francisella tularensis (F. tularensis), which is highly infectious and considered as a potential bioweapon. Currently there are four recognized subspecies of $F$. tularensis: subsp. tularensis, holarctica, mediasiatica and novicida. The former two subspecies are clinically important. F. tularensis is widely distributed in the Northern hemisphere and is capable of infecting hundreds of different vertebrates and invertebrates [1-3]. In addition, a variety of arthropods, including multiple genera of ticks as well as deer flies, fleas, mites, and mice, have been found to be naturally infected or experimentally competent as vectors. In many endemic 
areas, ticks play an important role in transmission of the disease from animals to humans [3], with the genera Amblyomma, Dermacentor, Haemaphysalis, Ixodes and Ornithodoros acting as main vectors [4].

In China, F. tularensis was isolated in Citellus dauricus from Tongliao region, Inner Mongolia municipality as early as in 1957 [5,6]. The first human tularemia epidemic, including 14 reported cases, which was caused by contact with infected hares, was reported in Heilongjiang province in 1959 [7]. The natural foci of the disease were subsequently identified in Tibet and Xinjiang municipalities [8-10]. The agent was detected and isolated from patients, Ixodex liberelis, Dermacentor marginatus and wolly hares (Lepus oiostolos) from Qinghai Province and Tibet, Xinjiang Autonomous Regions during 1962-1986 [8-11]. An outbreak of tularemia occurred in Shandong province in 1986. All the 31 reported patients were workers in a foodprocessing factory, where hares were slaughtered and processed [12]. Serological investigations demonstrated that the seropositive reaction to $F$. tularensis was significantly associated with tick exposure $[8,13]$. However, little is known about the prevalence of $F$. tularensis in ticks in China, lack of such information has inhibited our fully understanding of the disease ecology and also under-estimated the threat of the pathogen to human health where ticks are abundant. In the present study, we try to investigate the presence of F. tularensis in ticks, and then to identify their genotypes using multiple-locus variable-number tandem repeat analysis (MLVA).

\section{Results}

\section{Prevalence of F. tularensis in ticks}

Out of 1670 adult ticks examined, thirty three were positive for the fopA gene of $F$. tularensis. The overall positive rate was $1.98 \%$. All positive samples amplified the 220-bp fragment for the ppI-helicase gene that identifies F. tularensis subsp.holarctica (GenBank accession no. EU526911). Thus, all ticks in the present study were infected with $F$. tularensis subsp.holarctica. The positive rates of F. tularensis in D. silvarum were $3.67 \%$ and $2.33 \%$ in I. persulatus (Table 1), while all of the 144 Haemaphysalis verticalis and
299 Boophilus microplus were PCR-negative. The difference in positive rates among different species was significant $\left(\chi^{2}=14.366, P=0.002\right)$. We were able to detect $F$. tularensis in ticks from Jilin, Heilongjiang and Inner Mongolia, but not from Fujian during the present study. The positive rates varied according to their geographical origins (Table $1)$, with minor statistical significance $\left(\chi^{2}=7.865, P=\right.$ $0.049)$.

\section{MLVA}

Two F. tularensis MLVA markers (Ft-M3 and Ft-M10) were successfully amplified from the 33 samples that tested positive for both the fopA gene and PPI-helicase PCRs. The sequences from Ft-M10 were invariant, 2 copies and 361 bp in size (GenBank accession no. EU544140), same as previously reported [14]. The Ft-M3 loci had two allelic variants (9A: AACAAAGAC and 9E: AATAAGGAT), exactly as defined by Johansson for F. tularensis subsp. holarctica $[15,16]$. The sequence size from Ft-M3 ranged greatly from 270 to $369 \mathrm{bp}$ and the repeating units differed from 7 to 18 copies (GenBank accession no. EU544141 to EU544147). Based on the copy numbers of two loci, seven genotypes were identified (Table 2). The Ft-M3 genotype containing 9 repeat copies was detected at 3 study sites in over half of the F. tularensis positive ticks (17/33, $51.5 \%)$. The significance of this genotype-host association remains unknown. Different genotypes could be detected from the same location (Table 2), among which, one FtM3 genotype containing 7 repeat copies from $I$. persulcatus was unique which had been found existence in F. tularensis subsp. tularesnsis in Goethert's study [17].

\section{Discussion}

Although F. tularensis can be cultured in the laboratory, it requires enriched growth media and BSL3 facilities for isolation, these problems, together with the difficulty of recognizing the bacterium make it lack rapid and safe methods for laboratory identification [18]. Nowadays, various PCR approaches amplifying different genomic segments have been applied to detecting F. tularensis [1922 ]. The evaluations were performed during epidemics in Sweden in 1995 and 1998, respectively. It proved PCR

Table I: Detection for $F$. tularensis in ticks by species and geographic origins in China

\begin{tabular}{|c|c|c|c|c|c|}
\hline \multirow[t]{2}{*}{ Tick species } & \multicolumn{5}{|c|}{ No. positive/no. detected (\%) } \\
\hline & Jilin & Heilongjiang & Inner Mongolia & Fujian & Total \\
\hline Dermacentor sylvarum & $12 / 327(3.67)$ & --.-* & --- & --- & $12 / 327(3.67)$ \\
\hline Ixodes persulatus & $0 / 100(0)$ & $9 / 400(2.25)$ & $12 / 400(3.00)$ & --- & $21 / 900(2.33)$ \\
\hline Haemaphysalis verticalis & --- & -- & $0 / 144(0)$ & --- & $0 / 144(0)$ \\
\hline Boophilus microplus & --- & --- & --- & $0 / 299(0)$ & 0/299(0) \\
\hline Total & $12 / 427(2.8 \mid)$ & $9 / 400(2.25)$ & I2/544 (2.21) & $0 / 299(0)$ & $33 / 1670(1.98)$ \\
\hline
\end{tabular}

---, No ticks captured 
Table 2: Genotypes of $F$. tularensis detected from ticks in China

\begin{tabular}{|c|c|c|c|c|}
\hline \multirow[t]{2}{*}{ Geographical sites } & \multirow[t]{2}{*}{ Number } & \multirow[t]{2}{*}{ Hosts } & \multicolumn{2}{|c|}{ Genotypes for two marker } \\
\hline & & & Ft-M3 & Ft-MIO \\
\hline \multirow[t]{3}{*}{ Heilongjiang } & 1 & Ixodes persulcatus & 7 & 2 \\
\hline & 2 & I. persulcatus & 8 & 2 \\
\hline & 6 & I. persulcatus & 9 & 2 \\
\hline \multirow[t]{3}{*}{ Jilin } & 6 & Dermacentor sivarum & 9 & 2 \\
\hline & 2 & D.sivarum & 15 & 2 \\
\hline & 4 & D.sivarum & 16 & 2 \\
\hline \multirow[t]{4}{*}{ Inner Mongolia } & 5 & Ixodes persulcatus & 9 & 2 \\
\hline & 3 & I. persulcatus & 15 & 2 \\
\hline & 3 & I. persulcatus & 14 & 2 \\
\hline & I & I. persulcatus & 18 & 2 \\
\hline
\end{tabular}

detection of $F$. tularensis was more sensitive than culture [18]. In present study, nested-PCR method which targeting fopA gene of $F$. tualrensis $[17,23]$ was used to detect $F$. tularensis in ticks from four provinces of China. We were able to detect $F$. tualrensis in D. silvarum and I. persulcatus from three northern provinces of China. The prevalence in ticks was $1.98 \%$, which is comparable with the prevalence of $2.1 \%-2.8 \%$ in D. reticulates from European countries $[4,24]$. Actually, as early as 2004 , during an epidemical survey we have detected $F$. tularensis in rodents from these three provinces [25]. The present findings, combined with our previous detection result in rodents, adds evidence to our belief that tularemia may be only confined to the northern part of China. During our study, we were able to detect $F$. tularensis in D. silvarum and I. persulatus, whereas not from Haemaphysalis verticalis and Boophilus microplus. Moreover, the F. tularensis strains in China were mainly isolated from these two tick genera [8-10]. The principal tick vectors seemed only include the genus Dermacentor and Ixodes in China, because we have found no evidence that other tick genera was involved in F. tularensis to date.

For the first time, our study identified the infection of $F$. tularensis in ticks from Jilin Province. Actually, natural infection of $F$. tularensis in rodents had been recorded from same study sites in 2006 [25]. Geographically, Jilin Province is adjacent to Heilongjiang Province where first human tularemia cases were reported and natural focus was established as early as in 1958 [7]. The study sites from two provinces were close to each other and they have the same ecological environment. So it was not surprising that we were able to detect $F$. tularensis in ticks from Jilin Province. However, it is still too early to state that tularemia natural focus exists in Jilin Province because until now we have not obtained the isolate and there is no any case report.

Tandem repeat regions are known to be highly variable and successfully used for typing bacterial species, even for discriminating individual isolates [15,17,26-28]. During our study, two loci (Ft-M3 and Ft-M10) were used to measure the genetic diversity of $F$. tularensis detected in the study. The sequence of the 16-bp repeating unit of FtM10 was invariant, this result was consistent with our previous study results [14]. Perhaps there was only one variant from Ft-M10 locus among Chinese F. tularensis, although about 10 variants had been identified [30], among which 3 variants for $F$. tularensis subsp. holarctica were identified [15]. 7 variants were identified for 33 positive samples from Ft-M3 locus, among which one unique type containing 7 repeat copies was firstly identified in $F$. tularensis subsp. holarctica, this type had been identified from $F$. tularensis subsp. tularensis infected tick samples in Goethert's study [17]. According to the sequences, we are sure that the F. tularensis detected in present study can all be grouped as the F. tularensis subsp. holarctica (type B), not $F$. tularensis subsp. tularensis. The type containing 9 repeat copies from Ft-M3 locus seemed to be dominant in the detected ticks, actually in Goethert's study they had identified one genotype (also from Ft-M3 locus containing 10 repeat copies) only existing in ticks, which belonged to F. tularensis subsp.tularensis (type A). A variety of samples should be included to illuminate whether there are differences in various genotype among host ranges.

\section{Conclusion}

This study showed the role of two tick species in harboring the pathogen of tularemia in natural environment in China. In endemic foci where these genera ticks are abundant, public health officials and clinicians should be alert to the presence of tularemia, although it is not notifiable disease in China. In addition, MLVA results disclosed the genetic diversity of detected $F$. tularensis and identified one dominant genotype existing in ticks. Future work will involve more markers other than Ft-M3 and Ft-M10 detected in multiple-labs so to make comparisons with isolates of worldwide origin possible $[29,30]$. 


\section{Methods \\ Tick collection}

Adult ticks were collected from Inner Mongolia Autonomous Region, Heilongjiang Province, Jilin Province and Fujian Province. Ticks from Inner Mongolia Autonomous Region were collected in April 2005. Others were collected during April to June in 2006. The first three collection sites are forested highlands in northeastern China, belonging to Great Xing'an Mountains and Small Xing'an Mountains with an elevation 500-825 m. There are many marsh existing in above three regions. The main vegetation types are coniferophyte, and in marsh region, Tansy, reeds, and Artemisia are dominant. The temperature ranges from $40^{\circ} \mathrm{C}$ to $30^{\circ} \mathrm{C}$. The study site in Fujian Province is wooded foothills in southern China. The main vegetation is broadleaved plant. At least 5 different sites were sampled for each province. Ticks were collected by dragging a cloth over the vegetation and were stored alive until brought into laboratory. In the laboratory, ticks were examined morphologically and sorted by species, developmental stage under microscope. After species identification, tick specimens were stored at $-20^{\circ} \mathrm{C}$ for further DNA extraction.

\section{DNA extraction}

Ticks were processed individually. DNA extraction was performed by a modification of a previously described method [31]. Briefly, each tick was soaked in 70\% ethanol for 10 minutes, and then rinsed three times in sterile water. Then the tick was placed into microtube and mechanically disrupted with sterile scissors in $50 \mu \mathrm{l}$ of DNA extraction buffer (10 mM Tris, pH 8.0, 2 mM EDTA, $0.1 \%$ SDS, $500 \mu \mathrm{g}$ of proteinase $\mathrm{K} / \mathrm{ml}$ ). Each sample was incubated for $2 \mathrm{~h}$ at $56^{\circ} \mathrm{C}$ and then boiled at $100^{\circ} \mathrm{C}$ for 10 min to inactivate proteinase $\mathrm{K}$. After centrifugation, the supernatant was transferred to a fresh sterile microtubule and purified by extracting twice with an equal volume of phenol-chloroform before use. Then TE (10 mM Tris, $\mathrm{pH}$ 8.0, 2 mM EDTA) was added to dissolve DNA for PCR use.

\section{PCR amplification}

Samples were screened for evidence of $F$. tularensis by nested PCR targeting the fopA gene, as described previously $[17,23]$. All positive specimens for the fopA gene were re-tested to obtain subspecies confirmation by amplifying the $p p I$-helicase region of $F$. tularensis gene structure using the primer pair of C6 (AGGCGGAGATCTAGGAACCTTT, GenBank accession no. AF247642) and C8 (AGCCCAAGCTGACTAAAATCTTT, GenBank accession no. AF247685). This PCR yielded a variable-length amplicon due to a 30-bp deletion in $F$. tularensis subsp.holarctica: for F. tularensis subsp.holarctica, the amplicon was 220 bp (GenBank accession no. EU526911), and for F. tularensis subsp.tularensis, the amplicon was $250 \mathrm{bp}$ in length [15]. All the PCR products were separated by 3\% agarose gel electrophoresis, stained with ethidium bromide, and visualized under UV light. Negative controls and positive controls (Chinese first $F$. tularensis strain isolated from Citellus dauricus in 1957 from Inner Mongolia) were applied to ensure validation of the amplification. To minimize contamination, DNA extraction, the reagent setup, amplification, and agarose gel electrophoresis were performed in separate rooms. Amplicons from all PCR assays were sequenced to verify the validity of the PCR. For sequencing, amplicons were excised from the gels, purified through spin columns (Qiagen, Hilden, Germany) and sequenced on automated DNA sequencer (ABI PRISM 377, Perkin-Elmer, Foster City, USA). All sequencing primers were amplification primers which were read for both strands.

\section{Multiple-locus variable number tandem repeat analysis (MLVA)}

Using MLVA, two markers, Ft-M3 and Ft-M10 (previous designations SSTR9 and SSTR16, respectively) were used to identify the genetic diversity among positive ticks as previously described [14,15]. All amplicons obtained from two markers were sequenced on an automated DNA sequencer (ABI PRISM 377 Parkin-Elmer, Inc). The number of repeated units for each sample was counted. The genotype was defined simply as the number of repeats for Ft-M3 followed by that for Ft-M10.

\section{Nucleotide sequence accession numbers}

Nucleic acid sequences representative of each genotype and sequence from subspecies confirmation were deposited in GenBank under accession numbers EU526911, EU544140 to EU544147.

\section{Authors' contributions}

FZ and WL carried out the samples detection and MLVA analysis, participated in ticks sampling and drafted the manuscript, ZTX performed the statistical analysis and drafted the manuscript. HY, XMW and QMZ participated in ticks sampling. WCC conceived of the study, participated in its design, coordinated the field investigation and drafted the manuscript. All authors read and approved the final manuscript.

\section{Acknowledgements}

This work was supported by grants from Natural Science Foundation of China (30600506) and the National Science Fund for Distinguished Young Scholars (No. 30725032). We thank Mrs. Peggy Wagner from Emory University for critical review.

\section{References}

I. Ellis J, Oyston PCF, Green M, Titball RW: Tularemia. Clin Microbiol Rev 2002, 15:63I-646.

2. Petersen JM, Schriefer ME: Tularemia: emergence/re-emergence. Vet Res 2005, 36:455-467.

3. Tärnvik A, Berglund L: Tularemia. Eur Respir J 2003, 21:361-373. 
4. Gordon JR, Mclaughlin BG, Nitituthai S: Tularemia transmitted by ticks (Dermacentor andersoni) in Saskatchewan. Can J Copm Med I 983, 47:408-4II.

5. Yang L: The report on isolation of Francisella tularensis from Citellus dauricus. Chin J Epidemiol 1958, 2:4-10.

6. Sun YD, Zhang ZX, Zhao B: The identification of Francisella tularensis-like organism isolated from Inner Mongolia. Chin J Epidemiol 1958, 2:15-27.

7. Kang CG: An epidemiological investigation on the first epidemic of human tularemia in China. Chin J Epidemiol 1980, I:248-25I.

8. Guo CH, Zen QG, Wang SC, Shi TF, Wu FL, Jia MH: Preliminary study of tularemia in Tibet Autonomous Region. Chin J Epidemiol 1981, 2:176-181.

9. Zhou XR, Liu SK, Wang TX, Zhang GL, Ma DX: Preliminary investigation on tularemia in Ali area. Endemic Diseases Bulletin 1993, 8:73-77.

10. Li L, Kou ZM: Preliminary study of tularemia in Ta-cheng District of the Xinjiang Uygur Autonomous Region. Chin J Epidemiolog 1985, 6:20-22.

II. Wang WH, Zhu HJ, Shi XQ, Zhou AQ: The report of isolating Francisella tularensis from Lepus oiostorum in Qinghai Province. Chin J Epidemiol 1980, 4:245-247.

12. Pang ZC: Investigation of the first outbreak of tularemia in Shandong Peninsula. Zhonghua Liu Xing Bing Xue Za Zhi 1987, 5:261-263.

13. Liu ZJ, Wang DH, Zhang YZ, Su DS, Shi SZ, Luo YQ, Sun ZX: Seroepidemiological study on tularemia from Qilian Mountain. China public health 1995, 7:320.

14. Liu W, He J, Duan Q, Zhang PH, Zhao QM, Wu XM, Yang H, Cao WC: Genotyping of Francisella tularensis strains isolated from China by short-sequence tandem repeat region analysis. Chin J Microbiol Immunol 2003, 23:826-828.

15. Johansson A, Granssön I, Larsson P, Sjstedt A: Extensive allelic variation among Francisella tularensis strains in a short-sequence tandem repeat region. J Clin Microbiol 200I, 39:3140-3।46.

16. Johansson A, Farlow J, Larsson P, Dukerich M, Chambers E, BystorÖm M: Worldwide genetic relationship among Francisella tularensis isolates determined by multiple-locus variable-number tandem repeat analysis. J Bacteriol 2004, 186:5808-58|8.

17. Goethert HK, Shani I, Telford SR: Genotypic Diversity of Francisella tularensis infecting Dermacentor variabilis Ticks on Martha's Vineyard, Massachusetts. J Clin Microbiol 2004, 42:4968-4973.

18. Johansson A, Forsman M, SjÖstedt A: The development of tools for diagnosis of tularemia and typing of Francisella tularensis. APMIS 2004, I I 2:898-907.

19. Johansson A, Berglund L, Eriksson U, GÖransson I, Wollin R, Forsman M, et al.: Comparative analysis of PCR versus culture for diagnosis of ulceroglandular tularemia. J Clin Microbiol 2000, 38:22-26.

20. SjÖstedt A, Eriksson U, Berglund L, Tärnvik A: Detection of Francisella tularensis in ulcers of patients with tularemia by PCR. J Clin Microbiol 1997, 35: 1045-1048.

21. Higgins JA, Hubalek Z, Halouzka J, Elkins KL, SjÖstedt A, Shipley M, et al.: Detection of Francisella tularensis in infected mammals and vectors using a probe-based polymerase chain reaction. Am J Trop Med Hyg 2000, 62(2):3 10-8.

22. Emanuel PA, Bell R, Dang JL, McClanahan R, David JC, Bergess RJ, et al.: Detection of Francisella tularensis within infected mouse tissues by using a hand-held PCR thermocycler. J Clin Microbiol 2003, 41:689-693.

23. Fulop M, Leslie $D$, Titball $R$ : A rapid, highly sensitive method for the detection of Francisella tularensis in clinical samples using the polymerase chain reaction. Am J Trop Med Hyg 1996, 54:364-366.

24. Tärnvik A, Priebe HS, Grunow R: Tularemia in Europe: An Epidemiological Overview. Scand J Infect Dis 2004, 36:350-355.

25. Zhang F, Liu W, Chu MC, He J, Duan Q, Wu XM, Zhang PH, Zhao $\mathrm{QM}$, Yang $\mathrm{H}$, Xin ZT, Cao WC: Francisella tularensis in rodents, China. Emerg Infect Dis 2006, I 2(6):994-6.

26. Pourcel C, Andr-Mazzeaud F, Neubauer H, Ramisse F, Vergnaud G: Tandem repeats analysis for the high phylogenetic analysis of Yersinia pestis. BMC Microbiology 2004, 4:22-30.
27. Ramisse V, Houssu P, Hernandez E, Denoeud F, Hilaire V, Lisanti O: Variable number of tandem repeats in Salmonella enterica subsp. enterica for typing purposes. J Clin Microbiol 2004, 42:5722-5730.

28. Top J, Schhouls LM, Bonten MJM, Willems RJL: Multiple-locus variable-number tandem repeat analysis, a novel typing scheme to study the genetic relatedness and epidemiology of Enterococcus faecium isolates. J Clin Microbiol 2004, 42:4503-45II.

29. Farlow J, Wagner DM, Dukerich M, Stanley M, Chu M, Kubota K: Francisella tularensis in the United States. Emerg Infect Dis 2005, I I:I835-I84I.

30. Farlow J, Smith KL, Wong J, Abrams M, Lytle M, Keim P: Francisella tularensis Strain Typing Using Multiple-Locus VariableNumber Tandem Repeat Analysis. J Clin Microbiol 200I, 39:3186-3192.

31. Cao WC, Zhao QM, Zhang PH, Dumler JS, Zhang XT, Fang LQ: Granulocytic ehrlichiae in Ixodes persulcatus ticks from an area in China where Lyme disease is endemic. J Clin Microbiol 2000, 38:4208-4210.
Publish with Bio Med Central and every scientist can read your work free of charge

"BioMed Central will be the most significant development for disseminating the results of biomedical research in our lifetime. "

Sir Paul Nurse, Cancer Research UK

Your research papers will be:

- available free of charge to the entire biomedical community

- peer reviewed and published immediately upon acceptance

- cited in PubMed and archived on PubMed Central

- yours - you keep the copyright
BioMedcentral 